\title{
Characterization of genome-wide genetic variations between two varieties of tea plant (Camellia sinensis) and development of InDel markers for genetic research
}

\author{
Shengrui Liu ${ }^{1 \dagger}$, Yanlin An ${ }^{1+}$, Wei Tong ${ }^{1}$, Xiuju Qin² ${ }^{2}$ Lidia Samarina ${ }^{3}$, Rui Guo ${ }^{1}$, Xiaobo Xia ${ }^{1}$ and Chaoling Wei ${ }^{1 *}$ (D)
}

\begin{abstract}
Background: Single nucleotide polymorphisms (SNPs) and insertions/deletions (InDels) are the major genetic variations and are distributed extensively across the whole plant genome. However, few studies of these variations have been conducted in the long-lived perennial tea plant.

Results: In this study, we investigated the genome-wide genetic variations between Camellia sinensis var. sinensis 'Shuchazao' and Camellia sinensis var. assamica 'Yunkang 10', identified 7,511,731 SNPs and 255,218 InDels based on their whole genome sequences, and we subsequently analyzed their distinct types and distribution patterns. A total of $48 \mathrm{InDel}$ markers that yielded polymorphic and unambiguous fragments were developed when screening six tea cultivars. These markers were further deployed on 46 tea cultivars for transferability and genetic diversity analysis, exhibiting information with an average 4.02 of the number of alleles $(\mathrm{Na})$ and 0.457 of polymorphism information content (PIC). The dendrogram showed that the phylogenetic relationships among these tea cultivars are highly consistent with their genetic backgrounds or original places. Interestingly, we observed that the catechin/caffeine contents between 'Shuchazao' and 'Yunkang 10' were significantly different, and a large number of SNPs/InDels were identified within catechin/caffeine biosynthesis-related genes.

Conclusion: The identified genome-wide genetic variations and newly-developed InDel markers will provide a valuable resource for tea plant genetic and genomic studies, especially the SNPs/InDels within catechin/caffeine biosynthesis-related genes, which may serve as pivotal candidates for elucidating the molecular mechanism governing catechin/caffeine biosynthesis.
\end{abstract}

Keywords: Molecular markers, Genetic diversity, SNP, InDel, Catechin/caffeine biosynthesis, Camellia sinensis

\section{Background}

Tea is the most popular non-alcoholic beverage and possesses numerous crucial properties including attractive aroma, pleasant taste, and helpful and medicinal benefits [1-3]. The tea plant (Camellia sinensis (L.) O. Kuntze) is a perennial evergreen woody plant $(2 \mathrm{n}=2 \mathrm{x}=30)$ belonging to the section Thea of the genus Camellia in the family Theaceae $[4,5]$. Evidence is accumulating that the tea plant was originated from Yunnan Province in

\footnotetext{
* Correspondence: weichl@ahau.edu.cn

†'Shengrui Liu and Yanlin An contributed equally to this work.

'State Key Laboratory of Tea Plant Biology and Utilization, Anhui Agricultural

University, 130 Changjiang West Road, Hefei, China

Full list of author information is available at the end of the article
}

southwestern China [4-7]. Currently, cultivated tea plant varieties primarily belong to two groups, Camellia sinensis var. sinensis (CSS) and Camellia sinensis var. assamica (CSA), are extensively cultivated in tropical and subtropical regions around the world [6, 8]. Generally, CSS is a slower-growing shrub with a relatively higher cold-resistance capacity, while CSA is quick-growing with larger leaves and high sensitivity to cold climate [9]. With the successive release of two draft genome sequences, CSA 'Yunkang 10' [10] and CSS 'Shuchazao' [9], this plant is rapidly becoming another tractable experimental model for genetics and functional genomics research on tea trees. It is known that self- 
incompatibility and long-term allogamy contributed considerably to the highly heterogeneous and abundant genetic variation of tea plant $[11,12]$. Therefore, it is highly important to characterize genome-wide genetic variation between the two varieties.

Molecular markers, based on DNA polymorphisms, are useful and powerful tools for genetic and breeding research. Numerous molecular markers have been successfully developed and applied in genetic and genomic research in tea plant, such as restriction fragment length polymorphisms (RFLPs), amplified fragment length polymorphisms (AFLPs), random amplification of polymorphic DNAs (RAPDs), cleaved amplified polymorphic sequences (CAPS), inter-simple sequence repeats (ISSRs), and simple sequence repeats (SSRs) [12, 13]. With the rapid development of the high-throughput sequencing approaches, the thirdgeneration single nucleotide polymorphism (SNP) and insertion/deletion (InDel) markers are gradually becoming the most widely used molecular markers, demonstrating a promising future in plant genetic and breeding research.

SNPs are the most abundant genetic variations in most plant species, and the exploitation of SNP markers in single-copy regions is considerably easier than use of the other DNA markers [14-16]. InDel markers have practical value for those laboratories with limited resources, which also showed reliable transferability between distinct populations [14, 17, 18]. Both SNPs and InDels have been extensively applied for breeding programs and genetic studies including pedigree analysis, origin and evolutionary analysis, population structure and diversity analysis, construction of linkage maps, QTL mapping, and marker-assisted selection [14, 19-22]. Several studies have also reported the development and application of SNP/InDel markers in tea plant genetic studies. For instance, 16 expressed sequence tag (EST)-SNP based CAPS markers were developed and applied for tea plant cultivar identification [23]. A set of SNPs from EST databases was identified and verified [24]. Fang et al. (2014) validated 60 EST-SNPs, and constructed genetic relationships among tea cultivars and their specific DNA fingerprinting [25]. Based on specific locus amplified fragment sequencing (SLAF-seq), a total of 6042 SNP markers were validated and a final genetic map containing 6448 markers was constructed [26]. Through restriction site-associated DNA sequencing (RAD-Seq) approach, Yang et al. (2016) identified a vast number of SNPs from 18 cultivated and wild tea accessions, and found that 13 genes containing non-synonymous SNPs exhibited strong selective signals suggesting artificial selective footprints during domestication of these tea accessions [27]. By harnessing the two reference genomes, it is now suitable for identifying genome-wide SNPs/
InDels between them to guide rapid and efficient development of markers for high-resolution genetic analysis.

The whole genome sequences of tea trees can provide an elegant platform for identifying abundant genetic variation and developing many genetic markers. The completion of the two reference genome sequences is a notable advance for genetic and genomic studies and a basis for this study. The tea plant whole genome CSA 'Yunkang 10' was first reported based on the Illumina next-generation sequencing platform, producing a $\sim 3.02$ Gb genome assembly containing 37,618 scaffolds with N50 length of $449 \mathrm{~Kb}$ [10]. Subsequently, the genome assembly of CSS 'Shuchazao' was released by combined Illumina and PacBio sequencing platforms, yielding a $3.14 \mathrm{~Gb}$ genome assembly that consists of 36,676 scaffolds with N50 length of $1.39 \mathrm{Mb}$ [9]. In this study, several principal objectives were completed. Genome-wide genetic variation and distribution patterns were investigated. A number of polymorphic and stable InDel markers were developed, providing informative molecular markers for genetic and genomic studies. The catechin and caffeine contents of the two tea cultivars were detected, and SNPs/InDels within catechin/caffeine biosynthesis-related genes were characterized. The identified genome-wide genetic variations and newly developed InDel markers provide valuable resources for tea plant genetic and genomic studies, and the identification of SNPs/InDels within catechin/caffeine biosynthesisrelated genes can serve as important candidate loci for functional analysis.

\section{Results}

\section{Mapping of clean reads to the reference genome 'Shuchazao'}

CSS 'Shuchazao' has been observed to have significant differences in bud, leaf and budding flower size compared with CSA 'Yunkang 10' (Fig. 1). The completion of the two reference genome sequences ('Shuchazao' and 'Yunkang 10') is a notable advance for comparative genomic studies on tea plants in Thea section. Therefore, genome-wide genetic variations were identified between the two genome assemblies. After filtering the raw data, a total of $324,154,064$ clean reads from the CSA whole genome sequencing data were generated; these reads had a coverage depth of 10.4X the 'Yunkang 10' genome with a $100 \mathrm{bp}$ length and $43 \%$ GC content. Through alignment, a total of $317,878,025$ clean reads were mapped to the reference genome, accounting for $98.1 \%$ of total reads. The mapped clean reads contained two types of sequencing reads: pair-end and single-end reads. The former was predominantly type $(317,063,284$, $99.7 \%$ ), while single-end reads accounted for only $0.3 \%$ $(814,741$ clean reads). 


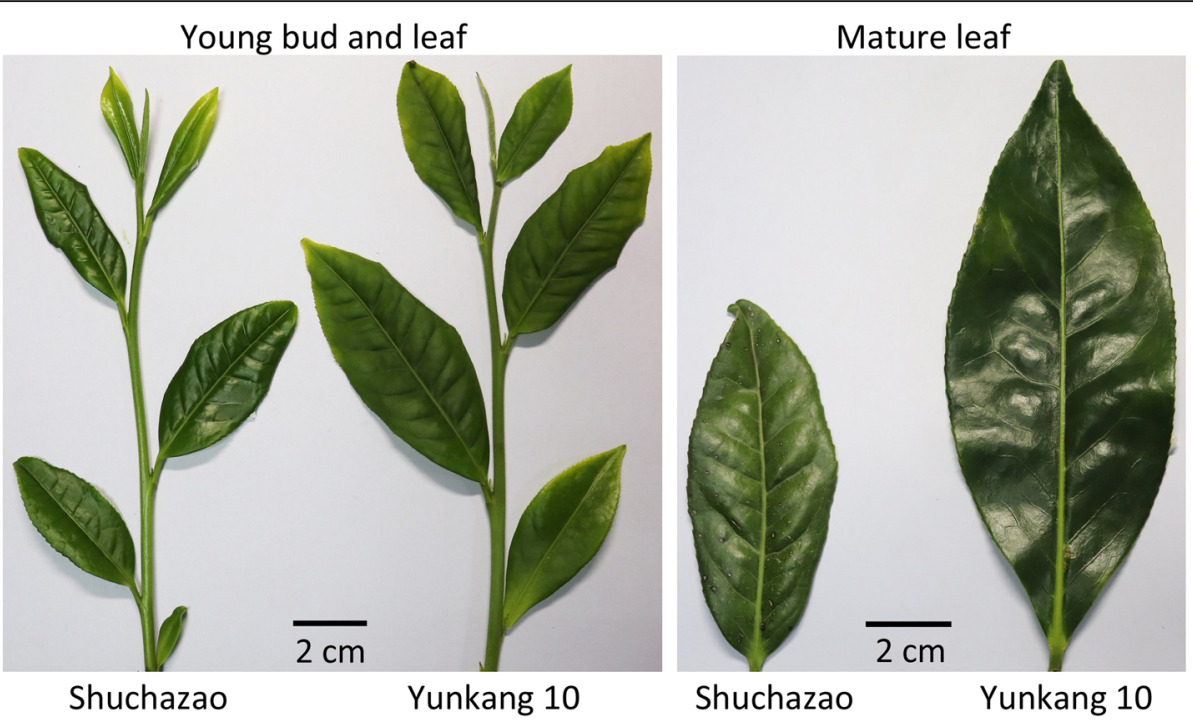

Fig. 1 Comparison of bud and leaf size between 'Shuchazao' and 'Yunkang 10'. Young buds and leaves were collected on April 2019, while mature leaves were collected from branches of last-year autumn
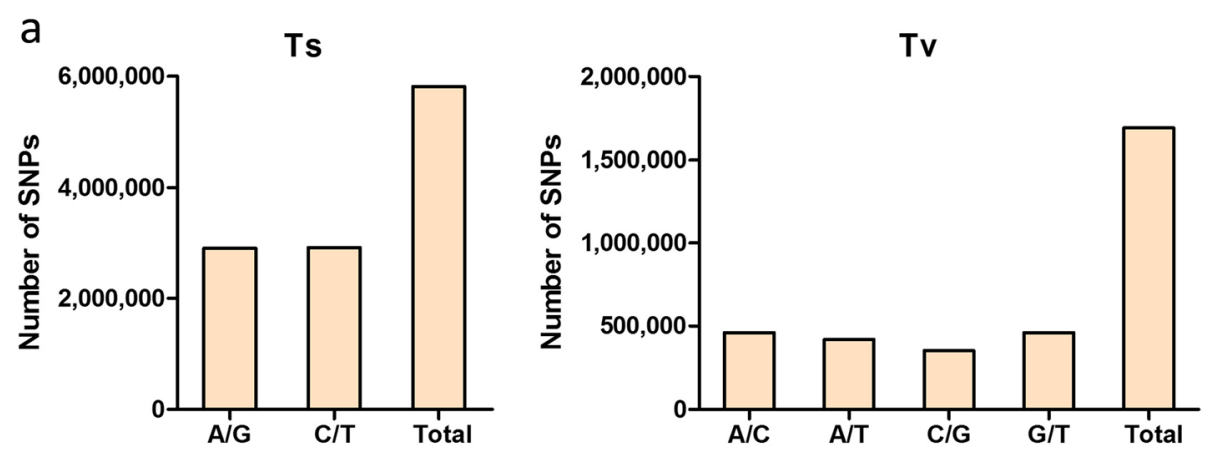

b

InDels

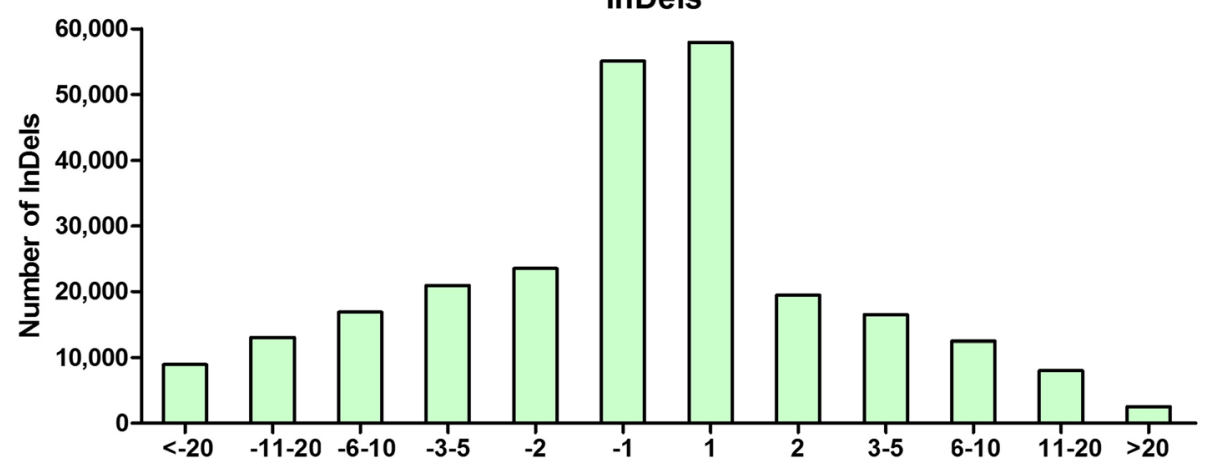

Fig. 2 Classification and distribution of identified SNPs/InDels in 'Yunkang 10'/ 'Shuchazao' comparison. a Frequency of different substitution types in the identified SNPs; the $x$-axis and y-axis represent the types and number of SNPs, respectively. $\mathbf{b}$ Distribution of the length of InDels identified between the two tea cultivars; the $x$-axis shows the number of nucleotides of InDels, and the $y$-axis represents the number of InDels at each length 


\section{Identification and distribution of SNP and InDel loci} After a series of filtering, a total of 7,071,433 SNP loci were generated, with an average SNP density in the tea genome being estimated to be $2341 \mathrm{SNPs} / \mathrm{Mb}$. Based on nucleotide substitutions, the detected SNPs were classified as transitions (Ts: G/A and $\mathrm{C} / \mathrm{T}$ ) and transversions (Tv: A/C, A/T, C/G, and G/T), which accounted for $77.46 \%(5,818,773)$ and $22.54 \%(1,692,958)$, respectively (Fig. 2a), with a $\mathrm{Ts} / \mathrm{Tv}$ ratio of 3.44. In transitions, the number of $\mathrm{A} / \mathrm{G}$ is equivalent to the $\mathrm{C} / \mathrm{T}$ type, which included 2,905,203 and 2,913,570, respectively. For transversions, the number of four types $(\mathrm{A} / \mathrm{C}, \mathrm{A} / \mathrm{T}, \mathrm{C} / \mathrm{G}$ and $\mathrm{G} / \mathrm{T}$ ) are almost evenly distributed with an insignificant difference among them, which accounted for $27.23 \%$ $(460,988), \quad 24.72 \% \quad(418,536), 20.84 \% \quad(352,802)$ and $27.21 \%(460,632)$, respectively (Fig. 2a).

A total of 255,218 InDels were identified, with an average density of $84.5 \mathrm{InDels} / \mathrm{Mb}$. The length distribution of InDels was analyzed by dividing the lengths into different groups and calculating the ratios for the corresponding length groups (Fig. 2b). It is obvious that mononucleotide InDels is the most abundant type, accounting for $44.27 \%(112,976)$ of the total number. The length of InDels ranging from 1 to 20 bp was predominant, accounting for more than $95.5 \%$ $(243,749)$ of the total InDels. A clear tendency was that the number of InDels gradually decreased with increasing InDel length.

\section{Location and functional annotation of SNPs and InDels}

The annotation of the 'Shuchazao' reference genome was used to uncover the distribution of SNPs and InDels within distinct genomic regions. According to the gene structure of the reference genome, the overwhelming number of SNPs (94\%) was identified in intergenic regions, while only $6 \%(440,298)$ of SNPs were located in genic regions (Fig. 3a). Among the SNPs located in genic regions, 89,511 SNPs were detected in the CDs region, which contained 38,670 synonymous and 50,841 nonsynonymous SNPs, respectively. Similarly, a small proportion of InDels were located in the genic regions, which accounted for only $12 \%(31,130)$ of the total number (Fig. 3b). Remarkably, 3406 InDels were located in the CDs region, which can be regarded as the preference for developing InDel markers.

To better understand the potential functions of these genetic variations within genes, GO term enrichment analysis of genes containing SNPs/InDels within CDs region was performed. These genes were classified into biological process, cellular component and molecular function categories (Additional file 2: Figure S2). Regarding the genes containing SNPs, the GO terms of cellular process, metabolic process and single-organism process were dominantly abundant in the biological process (Additional file 2: Figure S2A). In the cellular component category, the top three enriched GO terms were membrane, cell and cell part. Based on the molecular function category, catalytic activity and binding are predominantly enriched, while others accounted for a small proportion (Additional file 2: Figure S2A). Interestingly, a nearly consensus result was obtained for GO terms analysis of genes containing InDels, nothing but the number of genes is less compared with the number of genes containing SNPs (Additional file 2: Figure S2B).
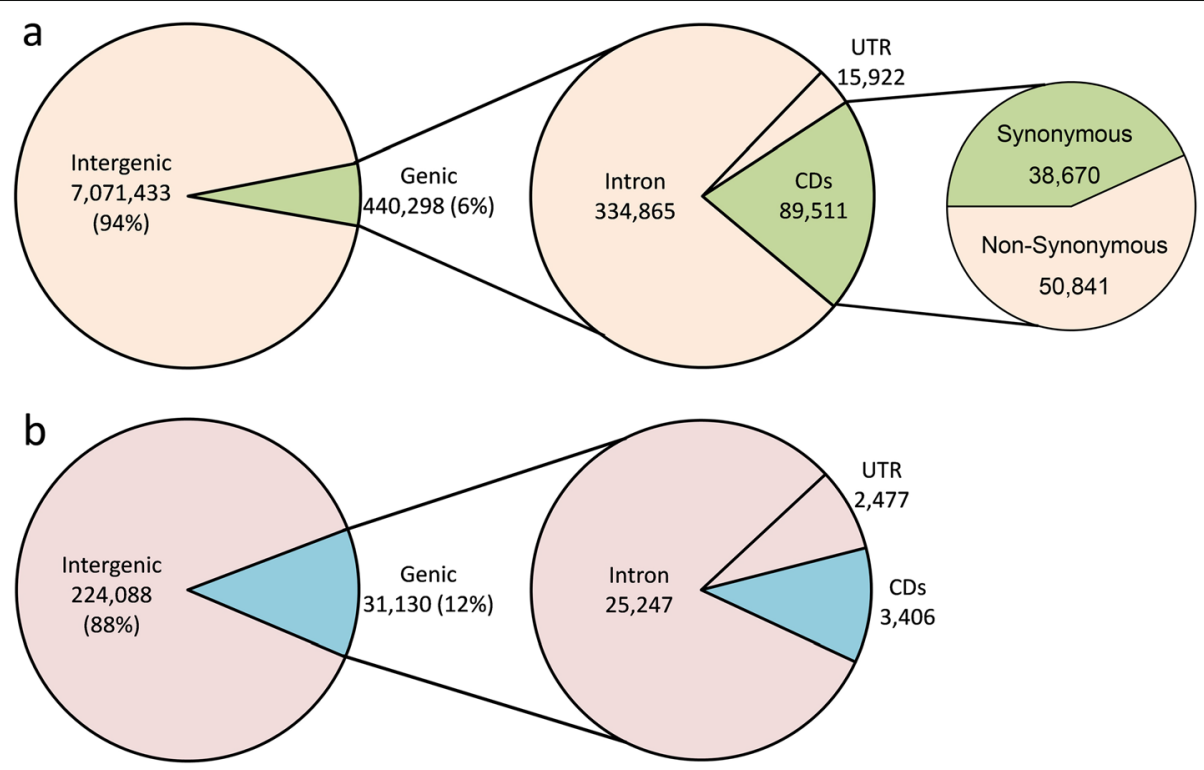

Fig. 3 Annotation of SNPs and InDels identified between 'Shuchazao' and 'Yunkang 10'. a Annotation of SNPs. b Annotation of InDels. SNPs and InDels were classified as intergenic and genic on the 'Shuchazao' reference genome, and locations within the gene models were annotated 
Validation and polymorphism of newly-developed InDel markers

Initially, all InDels were used for designing primer pairs using Primer3.0. To validate the InDels and develop polymorphic InDel markers, we selected 100 InDel markers that were distributed on different scaffolds. To facilitate the screening and development of more practical markers, the lengths of all selected InDels ranged from 5 to $20 \mathrm{bp}$ in length. To determine the reliability and polymorphisms of the primers, six tea cultivars were selected for testing their amplified fragments using Fragment Analyzer ${ }^{\mathrm{TM}}$ 96. Of the total primer sets tested, 48 primer pairs were successfully amplified with unambiguous bands and length polymorphisms among the six tea cultivars, 19 primer sets generated non-polymorphic or empty amplifications, and 33 primer pairs yielded nonspecific amplification or ambiguous bands.
Consequently, the 48 primer sets were regarded as elegant InDel markers and used for further analysis.

To test cross-cultivars/subspecies transferability, the 48 InDel markers were conducted on a panel of 46 tea cultivars belonging to section Thea of genus Camellia. The detailed information of the 46 tea cultivars is listed in Additional file 4: Table S1. The results of $18 \mathrm{InDel}$ markers testing on various tea cultivars are shown in Fig. 4, demonstrating that unambiguous and polymorphic bands were obtained based on these markers. The amplified results of the remaining 30 markers were also demonstrated (Additional file 3: Figure S3). For the newly developed markers, 20, 25 and 3 InDel markers generated high polymorphism, moderate polymorphism, and low polymorphism in the 46 tea cultivars, respectively. The PIC value of each InDel marker was presented in Table 1. The amplified allele sizes across them were

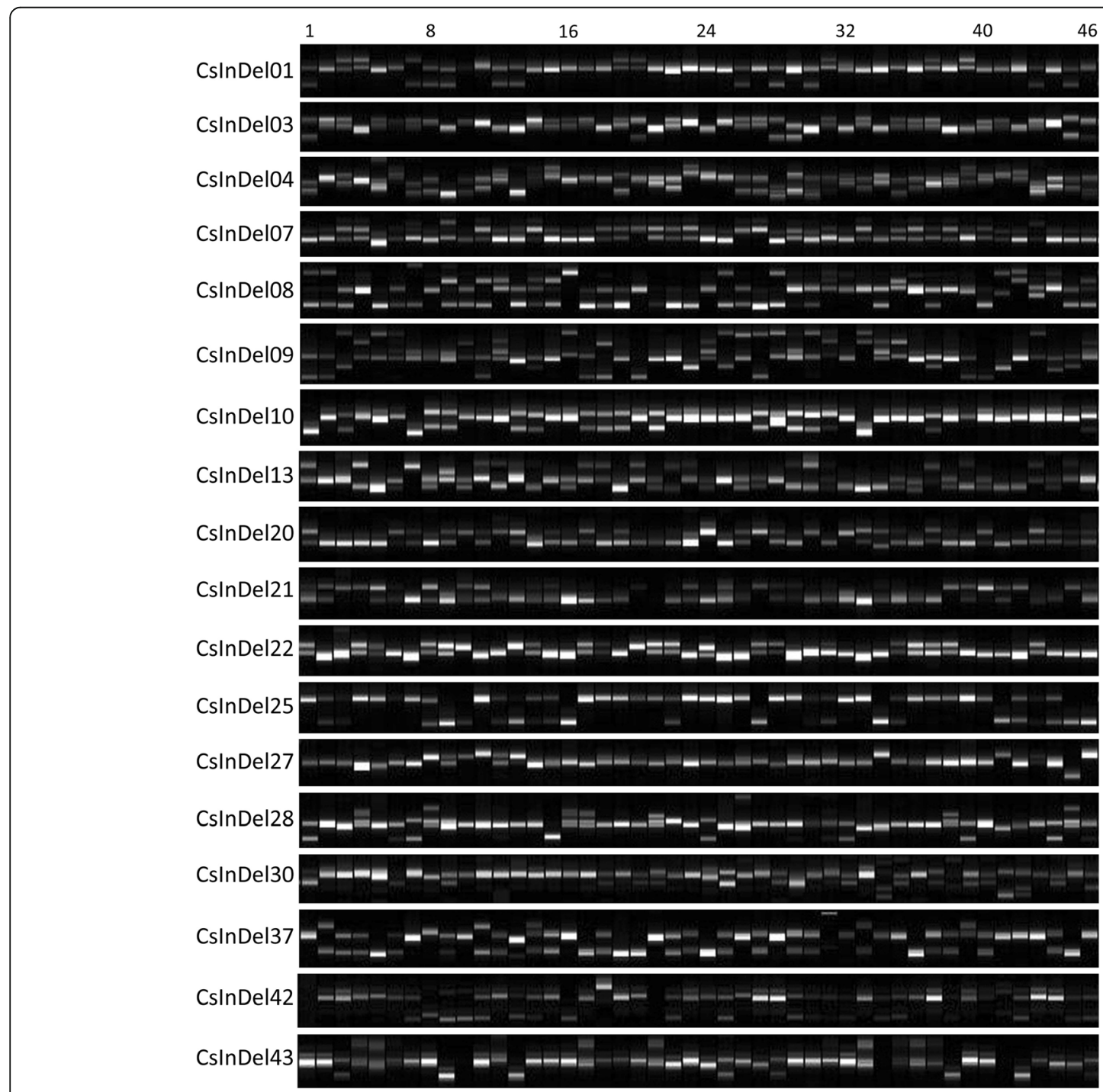

Fig. 4 Exhibition of transferability and polymorphism detected by 18 out of 48 InDel markers among 46 tea cultivars 
Table 1 Characteristics of 48 newly developed InDel markers

\begin{tabular}{|c|c|c|c|c|c|c|c|}
\hline Marker ID & Scaffold location & Fragment size (bp) & $\mathrm{Na}$ & MAF & Ho & $\mathrm{He}$ & PIC \\
\hline CslnDel01 & Scaffold 5: 236696 & $139-156$ & 3 & 0.787 & 0.383 & 0.361 & 0.327 \\
\hline CsInDel02 & Scaffold 5: 1208833 & $186-205$ & 4 & 0.489 & 1.000 & 0.633 & 0.555 \\
\hline CslnDel03 & Scaffold 12: 195263 & $332-354$ & 3 & 0.500 & 0.489 & 0.577 & 0.478 \\
\hline CslnDel04 & Scaffold 30: 3820588 & $214-242$ & 5 & 0.532 & 0.532 & 0.636 & 0.576 \\
\hline CsInDel05 & Scaffold 39: 128636 & $236-264$ & 4 & 0.479 & 0.979 & 0.556 & 0.448 \\
\hline CslnDel06 & Scaffold 41: 2074123 & $280-295$ & 3 & 0.808 & 0.180 & 0.319 & 0.273 \\
\hline CsInDel07 & Scaffold 46: 249178 & $176-189$ & 3 & 0.734 & 0.362 & 0.405 & 0.336 \\
\hline CslnDel08 & Scaffold 51: 314982 & $206-215$ & 6 & 0.394 & 0.638 & 0.691 & 0.627 \\
\hline CsInDel09 & Scaffold 51: 760768 & $201-248$ & 7 & 0.532 & 0.660 & 0.679 & 0.645 \\
\hline CslnDel10 & Scaffold 52: 469482 & $288-306$ & 3 & 0.745 & 0.255 & 0.394 & 0.329 \\
\hline CslnDel11 & Scaffold 60: 843530 & $292-332$ & 6 & 0.383 & 0.213 & 0.748 & 0.701 \\
\hline CslnDel12 & Scaffold 60: 843632 & $240-275$ & 5 & 0.426 & 0.660 & 0.704 & 0.645 \\
\hline CslnDel13 & Scaffold 64: 151635 & $270-289$ & 3 & 0.404 & 0.617 & 0.643 & 0.559 \\
\hline CsInDel14 & Scaffold 66: 500052 & $203-232$ & 4 & 0.436 & 0.064 & 0.621 & 0.535 \\
\hline CslnDel15 & Scaffold 77: 505984 & $185-207$ & 2 & 0.500 & 1.000 & 0.505 & 0.375 \\
\hline CslnDel16 & Scaffold 89: 1202911 & $231-248$ & 2 & 0.819 & 0.149 & 0.300 & 0.252 \\
\hline CslnDel17 & Scaffold 98: 664107 & $306-354$ & 6 & 0.395 & 0.256 & 0.731 & 0.677 \\
\hline CslnDel18 & Scaffold 114: 416691 & $283-326$ & 6 & 0.489 & 0.809 & 0.703 & 0.661 \\
\hline CslnDel19 & Scaffold 129: 540746 & $180-214$ & 6 & 0.422 & 1.000 & 0.652 & 0.579 \\
\hline CslnDel20 & Scaffold 154: 767901 & $285-297$ & 5 & 0.266 & 0.979 & 0.763 & 0.709 \\
\hline CsInDel21 & Scaffold 225: 80286 & $191-204$ & 2 & 0.649 & 0.362 & 0.461 & 0.352 \\
\hline CslnDel22 & Scaffold 1000: 52494 & $216-288$ & 3 & 0.532 & 0.404 & 0.612 & 0.537 \\
\hline CsInDel23 & Scaffold 1001: 123324 & $236-326$ & 6 & 0.628 & 0.489 & 0.568 & 0.526 \\
\hline CsInDel24 & Scaffold 1001: 149678 & 190-199 & 2 & 0.798 & 0.021 & 0.326 & 0.271 \\
\hline CslnDel25 & Scaffold 1001: 155681 & $195-218$ & 2 & 0.649 & 0.319 & 0.461 & 0.352 \\
\hline CsInDel26 & Scaffold 1001: 1251845 & $341-363$ & 3 & 0.583 & 0.833 & 0.511 & 0.399 \\
\hline CslnDel27 & Scaffold 1001: 1261469 & $273-290$ & 3 & 0.777 & 0.064 & 0.359 & 0.306 \\
\hline CslnDel28 & Scaffold 1001: 1400899 & $213-253$ & 6 & 0.660 & 0.383 & 0.537 & 0.501 \\
\hline CslnDel29 & Scaffold 1001: 1491192 & $182-226$ & 4 & 0.457 & 1.000 & 0.586 & 0.489 \\
\hline CslnDel30 & Scaffold 1001: 1691928 & $238-258$ & 4 & 0.745 & 0.362 & 0.411 & 0.363 \\
\hline CslnDel31 & Scaffold 1001: 1982826 & $284-316$ & 4 & 0.489 & 0.915 & 0.619 & 0.539 \\
\hline CslnDel32 & Scaffold 1452: 285463 & $272-299$ & 3 & 0.596 & 0.426 & 0.511 & 0.406 \\
\hline CsInDel33 & Scaffold 1539: 196438 & $271-280$ & 2 & 0.798 & 0.404 & 0.326 & 0.271 \\
\hline CslnDel34 & Scaffold 1541: 138532 & $265-286$ & 3 & 0.564 & 0.851 & 0.523 & 0.413 \\
\hline CslnDel35 & Scaffold 1543: 253456 & $172-207$ & 2 & 0.915 & 0.128 & 0.157 & 0.144 \\
\hline CsInDel36 & Scaffold 1551: 196819 & $157-237$ & 3 & 0.606 & 0.745 & 0.499 & 0.391 \\
\hline CslnDel37 & Scaffold 1553: 529121 & $211-237$ & 4 & 0.564 & 0.511 & 0.547 & 0.451 \\
\hline CsInDel38 & Scaffold 1555: 5209 & $109-340$ & 14 & 0.298 & 0.489 & 0.869 & 0.849 \\
\hline CslnDel39 & Scaffold 1579: 1466247 & $261-272$ & 2 & 0.606 & 0.787 & 0.483 & 0.363 \\
\hline CslnDel40 & Scaffold 1592: 672899 & $276-329$ & 7 & 0.596 & 0.979 & 0.666 & 0.489 \\
\hline CslnDel41 & Scaffold 1593: 1022219 & $172-187$ & 2 & 0.957 & 0.085 & 0.082 & 0.078 \\
\hline CsInDel42 & Scaffold 1594: 195199 & 184-206 & 3 & 0.691 & 0.426 & 0.454 & 0.380 \\
\hline CslnDel43 & Scaffold 1611: 1270988 & $226-254$ & 5 & 0.426 & 0.319 & 0.684 & 0.619 \\
\hline CslnDel44 & Scaffold 2220: 166816 & $292-328$ & 3 & 0.543 & 0.575 & 0.521 & 0.402 \\
\hline
\end{tabular}


Table 1 Characteristics of 48 newly developed InDel markers (Continued)

\begin{tabular}{llllllll}
\hline Marker ID & Scaffold location & Fragment size (bp) & Na & MAF & Ho & He \\
\hline CsInDel45 & Scaffold 15,285: 211487 & $281-321$ & 5 & 0.333 & 0.952 & 0.752 & 0.699 \\
CsInDel46 & Scaffold 15,433: 302840 & $190-253$ & 2 & 0.638 & 0.468 & 0.467 & 0.355 \\
CsInDel47 & Scaffold 15,579: 267174 & $176-186$ & 2 & 0.957 & 0.043 & 0.082 & 0.078 \\
CsInDel48 & Scaffold 15,650: 137667 & $228-266$ & 6 & 0.489 & 0.596 & 0.671 & 0.614 \\
Average & - & - & 4.02 & 0.585 & 0.524 & 0.528 & 0.457 \\
\hline
\end{tabular}

Na number of alleles, MAF major allele frequency, Ho observed heterozygosity, He expected heterozygosity, PIC polymorphism information content

within the ranges detected in the donor tea cultivar, implying that the amplified fragments were derived from the same loci and that the primer binding sites of the alleles were highly conserved among distinct tea cultivars/ subspecies. Several crucial parameters for evaluating polymorphism of markers were subsequently conducted, such as the number of alleles $(\mathrm{Na})$ per locus ranged from 2 (CsInDel15, CsInDel16, CsInDel21, CsInDel24, CsInDel25, CsInDel33, CsInDel35, CsInDel39, CsInDel41, CsInDel46, and CsInDel47) to 14 (CsInDel38) with an average of 4.02 alleles, the major allele frequency (MAF) ranged from the lowest 0.266 (CsInDel20) to the highest at 0.957 (CsInDel41 and CsInDel47) with an average of 0.585 , the observed heterozygosity $(\mathrm{Ho})$ ranged from 0.021 (CsInDel24) to 1.000 (CsInDel15, CsInDel19, and CsInDel29) with an average of 0.524 and the expected heterozygosity $(\mathrm{He})$ ranged from 0.082 (CsInDel41 and CsInDel47) to 0.869 with an average of 0.528 , the polymorphic information content (PIC) values were from the lowest value 0.078 (CsInDel41 and CsInDel47) to the highest 0.849 (CsInDel38) with an average of 0.457 (Table 1). Notably, the value of $\mathrm{He}$ has a similar variation trend as the PIC value, while it has a distinct variation trend with $\mathrm{Ho}$ values. The primer sequences and genomic locations of these newly developed markers are listed in Additional file 5: Table S2. These results showed that these newly developed InDel markers are informative and possess good transferability among various tea subspecies/cultivars.

\section{Population structure and genetic relationship analysis}

Population structure analysis was performed on the 46 tea cultivars using Structure 2.3.3 software based on 48 newly-developed InDel markers. The Q-plot output presented our grouping results, indicating that the two groups were the optimal classification at $\mathrm{K}=2$ (Fig. 5a). Apparently, tea cultivars from southern and southwestern China (Guangxi, Guangdong, Yunnan and Sichuan Provinces) belonging to Camellia sinensis var. assamica were clustered tightly together. In comparison, the tea cultivars possessing smaller leaf sizes and shorter heights that were cultivated in several other provinces were classified into another group (Fig. 5b).
To further confirm the applicability of the developed InDel markers for classification, we constructed a phylogenetic tree based on their genetic distances (Fig. 5c). Two major branches were generated (designated as $\alpha$ and $\beta$ groups), which contained 17 and 29 tea cultivars, respectively. Group $\alpha$ can be further divided into two subgroups, which were designated as $\alpha-1$ and $\alpha-2$ subgroups and consisted of 13 and 4 members, respectively. The dendrogram reflects that the phylogenetic relationships among them are highly consistent with their backgrounds or places of origin, as well as displaying consistency with the results from population structure analysis although a small discrepancy was observed (Fig. 5c).

\section{Identification of genetic variation in catechin/caffeine biosynthesis-related genes}

Tea cultivars belonging to Camellia sinensis var. assamica possess significant differences in phenotypes (plant height, leaf size and flower) and major characteristic secondary metabolites (such as catechin and caffeine, which contributed tremendously to tea quality) compared with Camellia sinensis var. sinensis. Therefore, we detected the contents of catechin (flavan-3-ols) and caffeine in both 'Shuchazao' and 'Yunkang 10' based on HPLC analysis. The total content of catechin in both buds and the second leaf from 'Yunkang 10' was higher than from 'Shuchazao' (Fig. 6a). To understand the potential molecular mechanism of difference, we performed the catechin biosynthesis pathway based on several previous studies (Fig. 6b). After search, we identified a number of SNPs and InDels in some crucial genes that are involved in the catechin biosynthesis pathway, including phenylalanine ammonia-lyase (PAL), cinnamic acid 4hydroxylase $(\mathrm{C} 4 \mathrm{H})$, 4-coumarate-CoA ligase $(4 \mathrm{CL})$, chalcone synthase (CHS), chalcone isomerase ( $\mathrm{CHI})$, flavanone 3-hydroxylase (F3H), flavonoid 3'-hydroxylase $\left(F^{\prime} H\right)$, flavonoid 3',5'-hydroxylase (F3'5'H), dihydroflavonol 4-reductase (DFR), leucoanthocyanidin reductase (LAR), anthocyanidin synthase (ANS), anthocyanidin reductase (ANR), and 1-O-galloyl- $\beta$-D-glucose Ogalloyltransferase (ECGT, which belongs to subclade 1A of serine carboxypeptidase-like (SCPL) acyltransferases) (Table 2). 

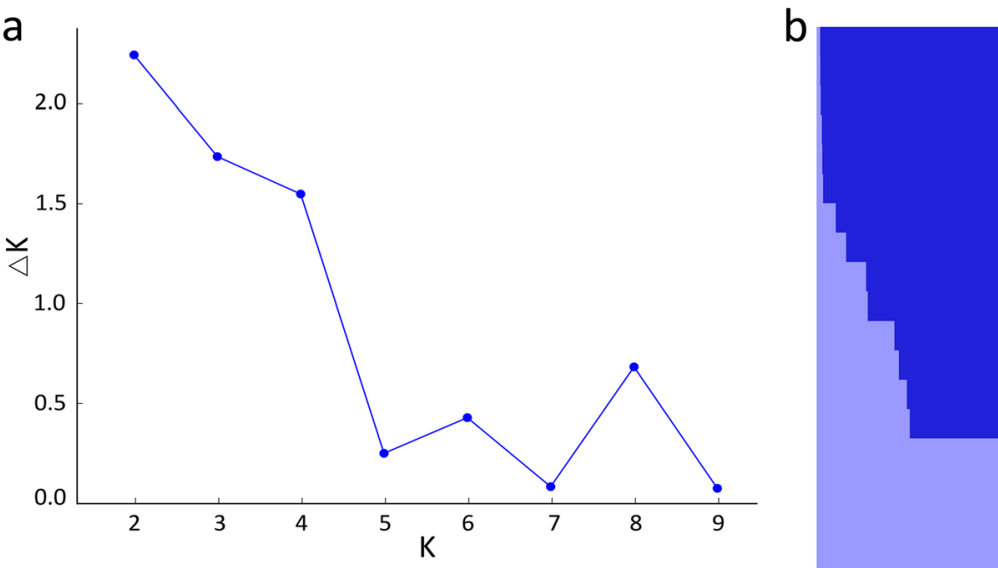

\begin{tabular}{|c|}
\hline Yinghong 9 \\
\hline Lianmei 601 \\
\hline Zihong \\
\hline Xueya 100 \\
\hline funkang 10 \\
\hline Zijuan \\
\hline Liubaoxiye \\
\hline Lingyun 2 \\
\hline Zixian \\
\hline Jianhexiangcha \\
\hline Shancha 1 \\
\hline Keke 1 \\
\hline Kiuhong \\
\hline Keke 2 \\
\hline Baihaozao \\
\hline Guihong 1 \\
\hline Chuanmu 28 \\
\hline Chuancha 2 \\
\hline Zhenong 113 \\
\hline uzao 2 \\
\hline ongjing 43 \\
\hline Duanjiebaihao \\
\hline $\begin{array}{l}\text { Fudingdabai } \\
\text { Huangguanyin }\end{array}$ \\
\hline Anjibaicha \\
\hline Taicha 1 \\
\hline Zhuyeqi \\
\hline Yuexipinxi 901 \\
\hline Fenghuangshuixian \\
\hline Hongyan 12 \\
\hline rieguanyin \\
\hline cha 1 \\
\hline /ungui \\
\hline Zimudan \\
\hline Huangkui \\
\hline Echa 5 \\
\hline Beidou \\
\hline Zhongcha 108 \\
\hline Huangshanbaicha 3 \\
\hline Guyuxiang \\
\hline Ziyan \\
\hline Zianmei 809 \\
\hline Nuyedancong \\
\hline Kiaoxianghong \\
\hline Taoyuandaye \\
\hline
\end{tabular}

C

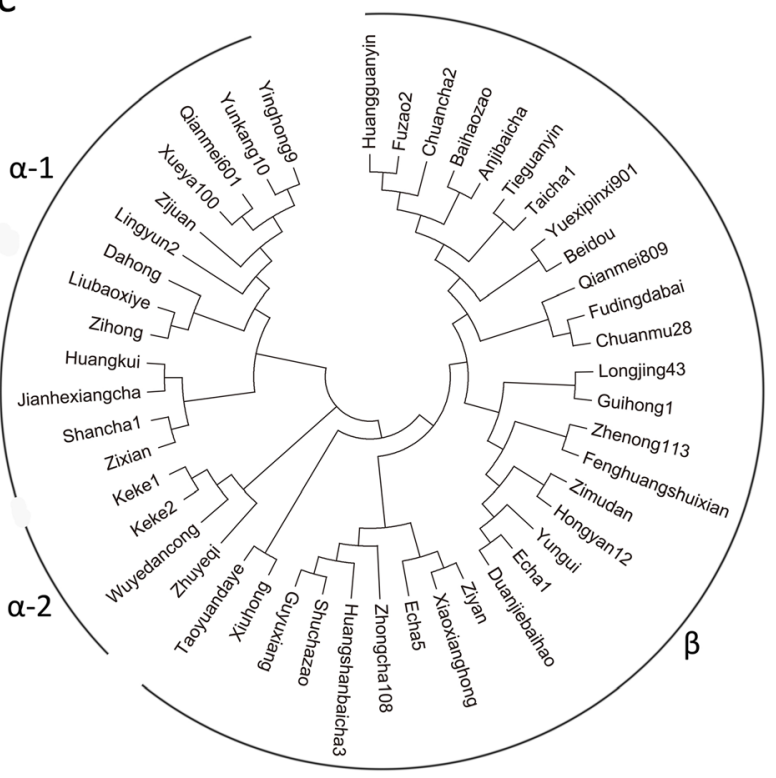

Shuchazao

Fig. 5 Population structure and phylogenetic relationship analysis based on 48 InDel markers. a Estimation of the optimal group number through $\Delta K$, the number of $K$ was set from 2 to 9 . $\mathbf{b}$ Q-plot of the population structure when $K=2$. Each tea cultivar is represented by a horizontal bar. $\mathbf{c}$ The dendrogram was constructed based on genotypes using neighbor-joining algorithm with 1000 bootstrap replicates

Detection of caffeine content in the two tea varieties demonstrated that the caffeine in both bud and the second leaf from 'Yunkang 10' is lower than that from 'Shuchazao' (Fig. 7a). In Fig. 7b, the wellstudied caffeine biosynthesis pathway was also performed based on previous studies [10, 28-31]. Similarly, a number of genetic variations within some critical regulatory genes were also detected, such as in IMP dehydrogenase (IMPDH), guanosine synthase (GMPS), $5^{\prime}$-nucleotidase $\left(5^{\prime}\right.$-Nase) and tea caffeine synthase (TCS) genes (Fig. 7c and Table 2). Collectively, these results indicate that certain genetic variations within these genes may explain the significant difference in catechin/caffeine synthesis between 'Shuchazao' and 'Yunkang 10'.

\section{Discussion}

\section{Identification of genetic variations in tea plant whole} genome

The recent release of the 'Shuchazao' and 'Yunkang 10' genome sequences will strongly facilitate the efficiency of comparative genomics and functional research in tea plants. This advance may enable researchers to study numerous agronomic traits associated with the perennial tea trees with a complete set of tools, including identification and development of SNP/InDel markers. Nevertheless, genome-wide identification and development of SNP/InDel markers are still in infancy, especially genetic variations related to important agronomical traits. By mapping the clean reads of 'Yunkang 10' to the reference genome assembly 'Shuchazao', we comprehensively 

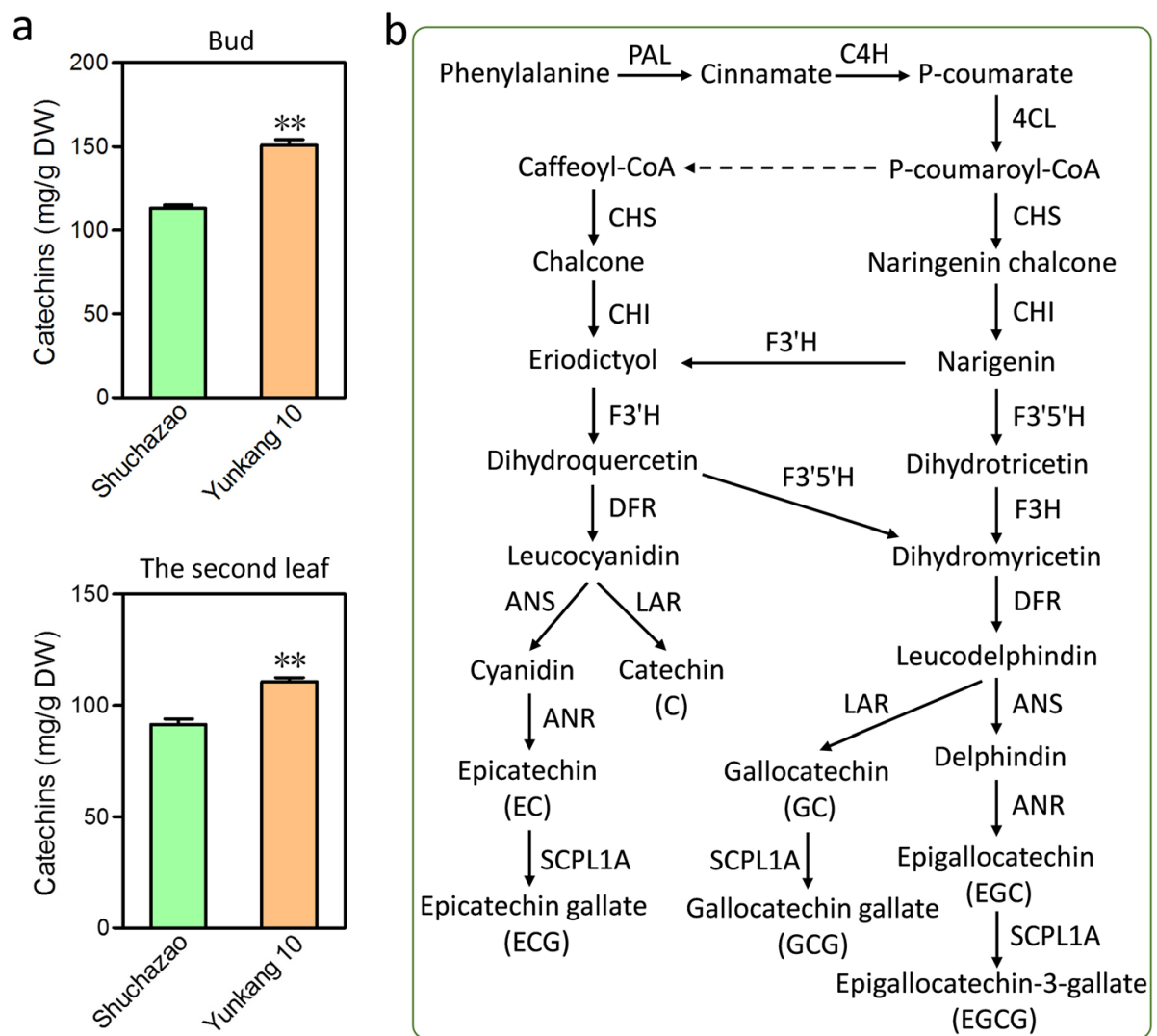

Fig. 6 Detection of catechin content and genetic variations within catechin biosynthesis-related genes. a Detection of catechin content of the bud and leaf of both 'Shuchaza' and 'Yunkang 10'. T-test was employed for significant analysis and two asterisks represent $p<0.01$. Each sample was tested with three independent biological replicates and two technical replicates. b The flavonoid biosynthesis pathway. PAL, phenylalanine ammonia-lyase; $\mathrm{C4H}$, cinnamic acid 4-hydroxylase; 4CL, 4-coumarate-CoAligase; $\mathrm{CHS}$, chalcone synthase; $\mathrm{CHI}$, chalcone isomerase; $\mathrm{F} 3 \mathrm{H}$, flavanone 3-hydroxylase; F3'H, flavonoid 3'-hydroxylase; F3',5'H, flavonoid 3',5'-hydroxylase; FLS, flavonol synthase; DFR, dihydroflavonol 4-reductase; ANS, anthocyanidin synthase; ANR, anthocyanidin reductase; LAR, leucocyanidin reductase; SCLP1A, subclade $1 \mathrm{~A}$ of serine

carboxypeptidase-like acyltransferases

surveyed DNA polymorphisms at the genome-wide scale and revealed the high level of genetic diversity between them. The vast number of SNPs and InDels identified in this study will provide valuable resources for tea plant genetics and breeding studies.

After filtering, a total of 7,071,433 SNPs and 255,218 InDels were identified, and their densities distributed in the tea plant genome were estimated to be 2341 SNPs/ $\mathrm{Mb}$ and $84.5 \mathrm{InDels} / \mathrm{Mb}$, respectively. The densities of SNP and InDel in the tea plant were significant differences compared with in other plant species, such as in Arabidopsis [32], Brassica rapa [17], quinoa [19], and soybean [33]. These significant differences in SNP/InDel density among different plant species may be due to the distinct filtering protocols and/or the different genomic composition. It is known that tea cultivars belonging to distinct varieties are highly heterogeneous with broad genetic variation due to their self-incompatibility and long-term allogamy [11]. In terms of SNPs, our results showed that $\mathrm{A} / \mathrm{G}$ and $\mathrm{C} / \mathrm{T}$ transitions are the most common pattern of nucleotide substitution, which is consistent with the results obtained in other plant species, such as foxtail millet [34], citrus [35], and soybean [33]. For InDels, the most prevalent types in the tea plant genome are short InDels. The number of $1-5 \mathrm{bp}$ InDels is the predominant types, accounting for $76 \%$ of all InDels, and similar results were displayed in several other plant species [14, 33-35].

Knowing the genomic positions of genetic variations in genetic markers or functional genes is highly important. It was shown that only minimal SNPs and InDels were distributed in the CDs region, which can be explained by the fact that the CDs region only accounted for a small proportion of the whole genome sequences and had relatively higher conservation compared with other regions. Among the 89,511 SNPs located in the CDs region, a total of 50,841 SNPs were nonsynonymous variations. Non-synonymous variations can usually have several functional impacts due to an altered amino acid sequence, such as hampering the interaction 
Table 2 Statistics on SNPS and InDels within catechin biosynthesis-related genes

\begin{tabular}{|c|c|c|c|c|c|c|c|c|c|c|c|}
\hline \multirow{2}{*}{$\begin{array}{l}\text { Gene } \\
\text { name }\end{array}$} & \multirow[t]{2}{*}{ Gene ID } & \multicolumn{2}{|l|}{ SNP } & \multicolumn{2}{|l|}{ InDel } & \multirow{2}{*}{$\begin{array}{l}\text { Gene } \\
\text { name }\end{array}$} & \multirow[t]{2}{*}{ Gene ID } & \multicolumn{2}{|l|}{ SNP } & \multicolumn{2}{|l|}{ InDel } \\
\hline & & DNA & CDs & DNA & CDs & & & DNA & CDs & DNA & CDs \\
\hline \multirow[t]{6}{*}{ PAL } & TEA014056.1 & 2 & 2 & 0 & 0 & $\mathrm{~F} 3 \mathrm{H}$ & TEA004906.1 & 0 & 0 & 1 & 0 \\
\hline & TEA034008.1 & 6 & 6 & 0 & 0 & & TEA010326.1 & 1 & 1 & 0 & 0 \\
\hline & TEA003137.1 & 16 & 16 & 0 & 0 & & TEA032907.1 & 3 & 3 & 1 & 1 \\
\hline & TEA023243.1 & 3 & 3 & 0 & 0 & & TEA028622.1 & 75 & 1 & 3 & 0 \\
\hline & TEA024587.1 & 3 & 3 & 0 & 0 & & TEA009737.1 & 4 & 4 & 0 & 0 \\
\hline & TEA003374.1 & 2 & 2 & 1 & 0 & & TEA000753.1 & 1 & 1 & 0 & 0 \\
\hline \multirow[t]{3}{*}{$\mathrm{C} 4 \mathrm{H}$} & TEA034001.1 & 16 & 8 & 1 & 1 & & TEA023937.1 & 1 & 1 & 0 & 0 \\
\hline & TEA016772.1 & 5 & 1 & 1 & 0 & & TEA016601.1 & 4 & 2 & 1 & 0 \\
\hline & TEA034002.1 & 6 & 6 & 0 & 0 & & TEA023790.1 & 10 & 3 & 1 & 0 \\
\hline \multirow[t]{12}{*}{$4 C L$} & TEA018887.1 & 1 & 1 & 0 & 0 & & TEA000474.1 & 8 & 1 & 0 & 0 \\
\hline & TEA034012.1 & 9 & 4 & 1 & 1 & & TEA026443.1 & 1 & 1 & 0 & 0 \\
\hline & TEA019275.1 & 14 & 10 & 0 & 0 & & TEA004898.1 & 1 & 1 & 0 & 0 \\
\hline & TEA027829.1 & 12 & 3 & 1 & 0 & & TEA006643.1 & 15 & 15 & 0 & 0 \\
\hline & TEA025906.1 & 2 & 1 & 0 & 0 & & TEA014951.1 & 29 & 8 & 2 & 0 \\
\hline & TEA009431.1 & 42 & 10 & 4 & 2 & DFR & TEA032730.1 & 2 & 0 & 1 & 0 \\
\hline & TEA018045.1 & 22 & 3 & 4 & 0 & & TEA023829.1 & 13 & 1 & 0 & 0 \\
\hline & TEA006577.1 & 6 & 1 & 0 & 0 & & TEA021807.1 & 2 & 0 & 0 & 0 \\
\hline & TEA031627.1 & 11 & 8 & 0 & 0 & & TEA021815.1 & 2 & 2 & 0 & 0 \\
\hline & TEA022274.1 & 2 & 1 & 0 & 0 & ANS & TEA010322.1 & 1 & 1 & 0 & 0 \\
\hline & TEA010681.1 & 8 & 4 & 0 & 0 & & TEA015762.1 & 1 & 1 & 0 & 0 \\
\hline & TEA002100.1 & 13 & 0 & 1 & 0 & & TEA015769.1 & 1 & 0 & 0 & 0 \\
\hline \multirow[t]{12}{*}{$\mathrm{CHS}$} & TEA018665.1 & 1 & 1 & 0 & 0 & ANR & TEA030023.1 & 1 & 1 & 0 & 0 \\
\hline & TEA034046.1 & 34 & 10 & 0 & 0 & & TEA022960.1 & 6 & 2 & 0 & 0 \\
\hline & TEA034011.1 & 6 & 4 & 0 & 0 & & TEA007646.1 & 1 & 0 & 1 & 0 \\
\hline & TEA034045.1 & 1 & 1 & 0 & 0 & & TEA003247.1 & 1 & 1 & 0 & 0 \\
\hline & TEA023331.1 & 2 & 2 & 0 & 0 & LAR & TEA021535.1 & 1 & 1 & 0 & 0 \\
\hline & TEA023340.1 & 3 & 3 & 2 & 0 & & TEA027582.1 & 0 & 0 & 2 & 0 \\
\hline & TEA034013.1 & 2 & 2 & 0 & 0 & & TEA009266.1 & 3 & 3 & 1 & 0 \\
\hline & TEA034043.1 & 31 & 7 & 0 & 0 & SCPLA1 & TEA034031.1 & 4 & 2 & 0 & 0 \\
\hline & TEA034019.1 & 3 & 3 & 0 & 0 & & TEA034032.1 & 11 & 5 & 0 & 0 \\
\hline & TEA034014.1 & 1 & 1 & 0 & 0 & & TEA010715.1 & 6 & 5 & 0 & 0 \\
\hline & TEA011908.1 & 6 & 1 & 0 & 0 & & TEA034056.1 & 33 & 1 & 0 & 0 \\
\hline & TEA019029.1 & 4 & 4 & 0 & 0 & & TEA009664.1 & 4 & 0 & 0 & 0 \\
\hline \multirow[t]{3}{*}{$\mathrm{CHI}$} & TEA034003.1 & 10 & 2 & 1 & 0 & & TEA016469.1 & 2 & 0 & 0 & 0 \\
\hline & TEA033023.1 & 127 & 4 & 10 & 0 & & TEA016463.1 & 9 & 1 & 0 & 0 \\
\hline & TEA033031.1 & 2 & 1 & 0 & 0 & & TEA034055.1 & 59 & 1 & 0 & 0 \\
\hline \multirow[t]{3}{*}{$\mathrm{F} 3^{\prime} \mathrm{H}$} & TEA016718.1 & 2 & 2 & 0 & 0 & & TEA034034.1 & 4 & 0 & 0 & 0 \\
\hline & TEA010133.1 & 5 & 2 & 0 & 0 & & TEA034036.1 & 1 & 1 & 0 & 0 \\
\hline & TEA006847.1 & 14 & 10 & 1 & 1 & & TEA023444.1 & 3 & 0 & 0 & 0 \\
\hline \multirow[t]{3}{*}{$\mathrm{F}^{\prime} 5^{\prime} \mathrm{H}$} & TEA013315.1 & 12 & 12 & 0 & 0 & & TEA034039.1 & 31 & 2 & 0 & 0 \\
\hline & TEA034021.1 & 6 & 1 & 0 & 0 & & TEA023451.1 & 4 & 1 & 0 & 0 \\
\hline & TEA034051.1 & 32 & 4 & 4 & 0 & & TEA000223.1 & 4 & 0 & 0 & 0 \\
\hline
\end{tabular}



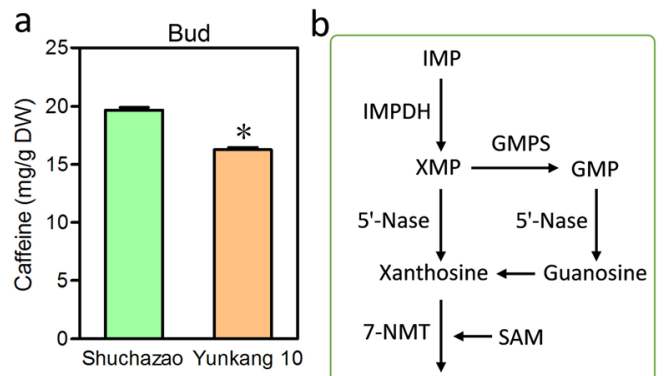

\begin{tabular}{|c|c|c|c|c|c|}
\hline \multirow{2}{*}{$\begin{array}{l}\text { Gene } \\
\text { name }\end{array}$} & \multirow[t]{2}{*}{ Gene ID } & \multicolumn{2}{|c|}{ SNP } & \multicolumn{2}{|c|}{ InDel } \\
\hline & & DNA & CDs & DNA & CDs \\
\hline \multirow[t]{2}{*}{ IMPDH } & TEA025393.1 & 105 & 2 & 0 & 0 \\
\hline & TEA028111.1 & 60 & 3 & 2 & 0 \\
\hline \multirow[t]{4}{*}{ GMPS } & TEA003403.1 & 26 & 1 & 2 & 0 \\
\hline & TEA006444.1 & 3 & 0 & 0 & 0 \\
\hline & TEA031622.1 & 18 & 7 & 3 & 1 \\
\hline & TEA011157.1 & 6 & 4 & 0 & 0 \\
\hline \multirow[t]{7}{*}{ 5'-Nase } & TEA004804.1 & 3 & 0 & 0 & 0 \\
\hline & TEA005058.1 & 17 & 1 & 3 & 0 \\
\hline & TEA011804.1 & 14 & 3 & 2 & 0 \\
\hline & TEA023055.1 & 28 & 1 & 4 & 0 \\
\hline & TEA025000.1 & 10 & 7 & 3 & 1 \\
\hline & TEA025001.1 & 11 & 3 & 0 & 0 \\
\hline & TEA027082.1 & 19 & 4 & 2 & 0 \\
\hline \multirow[t]{9}{*}{ TCS } & TEA015176.1 & 6 & 1 & 2 & 0 \\
\hline & TEA010054.1 & 26 & 2 & 1 & 0 \\
\hline & TEA028050.1 & 3 & 2 & 0 & 0 \\
\hline & TEA024636.1 & 2 & 2 & 0 & 0 \\
\hline & TEA030024.1 & 2 & 0 & 0 & 0 \\
\hline & TEA010123.1 & 71 & 2 & 1 & 0 \\
\hline & TEA012581.1 & 1 & 1 & 0 & 0 \\
\hline & TEA028049.1 & 1 & 1 & 0 & 0 \\
\hline & TEA028052.1 & 1 & 1 & 0 & 0 \\
\hline
\end{tabular}

Fig. 7 Detection of caffeine content and genetic variations within caffeine biosynthesis-related genes. a. Detection of catechin content of the bud and leaf of both 'Shuchaza' and 'Yunkang 10'. T-test was employed for significant analysis and one asterisk represents $p<0.05$. Each sample was tested with three independent biological replicates and two technical replicates. $\mathbf{b}$. The caffeine biosynthesis pathway. IMP, Inosine monophosphate; XMP, Xanthosine monophosphate; GMP, Guanosine monophosphate; IMPDH, IMP dehydrogenase; GMPS, Guanosine synthase; 5'-Nase, 5'-nucleotidase; 7-NMT, 7-methylxanthosine synthase; SAM, S-Adenosyl-L-methionine; N-MeNase, N-methylnucleotidase; TCS, tea caffeine synthase. c. SNPs and InDels calling in caffeine biosynthesis-related genes

between proteins and affecting gene expression due to the functional consequences of distinct motif binding at variation sites $[33,36]$. It is worth noting that a total of 3406 identified InDels were located in the CDs region. InDels tend to have more impact on protein structure and function than single base changes, especially those in the CDs region [33]. Nevertheless, genetic variations at UTRs may also play important roles, such as modification of regulatory elements affecting the interaction of the UTRs with proteins and miRNAs [37]. Overall, these SNPs and InDels can serve as important candidates for functional research, especially those InDels in the CDs, which can be considered as a valuable resource for developing phylogenetic and/or functional markers.

\section{Development and application of InDel markers}

Molecular markers are becoming indispensable tools for evolutionary analysis, germplasm identification and conservation, and marker-assisted selection (MAS). SSR is an extensively used marker type among genetic markers, and a large number of highly polymorphic SSR markers have been developed and applied in various genetic studies in tea plants $[8,13]$. These SSR markers, however, could easily result in non-specific amplifications and cause confusion in genotyping scoring [19], especially for plant species with large genome and high repetitive sequences. In fact, InDel markers are also PCR-based markers and are similarly affected by genomic complexity. However, they gave relatively less stutter bands due to the variations are more conservative compared with SSR markers $[18,19]$. Through a series of screenings, we developed a final of 48 polymorphic and stable InDel markers with 5-20 bp in length based on the genomic assembled sequences (Table 1). The length of fragments of the alleles amplified across tea cultivars was consistent with the expected sizes of the products, implying that the primer binding sites of the alleles were highly conserved. The large proportion of InDel markers displayed a moderate PIC value $(0.25<\mathrm{PIC}<0.5)$, and the average of PIC was 0.4. It is obvious that the PIC values of most InDel markers were lower than the PIC of the majority SSR markers [2, 8, 38, 39], supporting that the InDel markers are stable and bi-allelic throughout the genome. Therefore, these newly developed InDel markers are suitable for germplasm identification and conservation, genetic diversity analysis, population structure and phylogenetic relationship analysis. In addition, InDels can affect gene functions by causing the gain or loss of a frameshift and/or a stop codon, it is therefore suitable for developing functional markers that might be particularly valuable for MAS $[19,40]$.

Population structure analysis and phylogenetic trees can reflect the genetic diversity, pedigree relationships, and geographic distances among plant species and/or varieties $[2,16,22]$. They can also be used to evaluate the reliability of molecular markers. To test the reliability and practicability of the newly-developed InDel markers, population structure and phylogenetic 
relationship analysis were employed, and a consistent result was established (Fig. 5). Apparently, the tea cultivars from southern and southwestern China were clustered together, which originated from C. sinensis var. assamica populations. In comparison, most tea cultivars from central China had relatively close relationships with each other, which have distinct phenotypes, including small leaf size and short height of tea trees. These results indicate that the population structure analysis and phylogenetic tree reflect the relationships of the 46 tea cultivars, demonstrating the high reliability of these InDel markers for genetic analysis.

\section{Genetic variations within catechin/caffeine biosynthesis- related genes}

Catechin and caffeine are among the most important components in tea plant leaves, which enormously affect the quality of tea products and pharmacy [9, 41]. It is well-known that the contents of catechin and caffeine are influenced by genotypic factors, and significant differences can be observed among distinct tea varieties/ cultivars [31, 42, 43].

Based on HPLC detection, we found that the total catechin content from 'Yunkang 10' was significantly higher than that from 'Shuchazao' in both bud and the second leaf (Fig. 6a). Evidence has shown that the total catechin content of tea varieties tended to decline from the southern to the northern regions [42, 43], and our result is consistent with this tendency. Because catechins are important factors for the oxidation degree and dark tea was produced with severe fermentation during processing $[41,43]$, our results supported the fact that most tea cultivars belonging to Camellia sinensis var. assamica are more suitable for producing dark tea. To understand the potential molecular mechanisms, genetic variations within key genes associated with the catechin biosynthesis pathway were investigated between the two varieties. Unsurprisingly, a large number of SNPs and InDels were identified and some of them were located in the CDs (Table 2). Combining the results of detection of catechin constitutes, it is likely to successfully select certain candidate genetic variations associated with the genotypic factors. For instance, a study reported that a number of candidate allelic variants relating to catechin traits at the F3'5' $\mathrm{H}$ locus were identified, and the genetic effects of SNP840/848 were the most robust among them [41].

The result of HPLC detection showed that the caffeine content from 'Yunkang 10' was significantly lower than from 'Shuchazao' (Fig. 7a). Remarkably, a number of SNPs and InDels were found within some genes associated with the caffeine biosynthesis pathway (Fig. 7c). Previously, a study reported that a $252 \mathrm{bp}$ InDel mutation in the 5'-UTR of TCS1 plays a crucial role in caffeine biosynthesis [44]. Thus, our results can provide valuable candidates for identifying variations within genes related to caffeine biosynthesis. Overall, these valuable resources can be used for further validation, such as functional characterization, association analysis, or development of functional markers for markerassisted selection.

\section{Conclusions}

Comparison of the whole genome sequences between 'Yunkang 10' and 'Shuchazao' revealed a large amount of genetic variations, including SNPs and InDels, demonstrating that the tea plant genome is highly variable. The types of SNPs and InDels were subsequently investigated, and their distributions and annotations were also analyzed. Based on these InDel loci, a total of 48 novel InDel markers with moderate polymorphism and high stability were developed. Population structure and phylogenetic relationship analyses were conducted based on these markers, revealing that tea cultivars from Camellia sinensis var. assamica were apparently clustered together, while the other tea cultivars from Camellia sinensis var. sinensis were clustered into another group. Remarkably, significant differences were observed in catechin and caffeine content between 'Yunkang 10' and 'Shuchazao', and a number of SNPs and InDels were identified within genes related to the catechin/caffeine biosynthesis pathways.

\section{Methods}

\section{Plant materials and DNA extraction}

A total of 46 clonal tea cultivars were collected from the main tea-growing regions in China, and we obtained permission to collect all the tea samples. The details of these samples, including cultivar name, subspecies, germplasm type, registration number in China and cultivation region are listed in Additional file 4: Table S1. Two individuals ('Keke 1' and 'Keke 2') were collected from the local natural population in Guangdong Province with the local government's permission; three clonal tea cultivars ('Liubaoxiye', 'Lingyun 2' and 'Zihong') were collected from the Tea Germplasm Repository of the Tea Research Institute of Guangxi Province with permission; the rest of 41 clonal tea cultivars were commercial cultivars and cultivated widespread in China, which were deposited in the Tea Plant Cultivar and Germplasm Resource Garden in Guohe Town (N31 ${ }^{\circ} 9^{\prime}$, E117 $13^{\circ}$, Hefei, China) of our Institute (Anhui Agricultural University). Until now, a total of 107 national tea cultivars (NTCs) and 139 provincial tea cultivars (PTCs) were registered in China [45]. In this study, 20 NTCs and 13 PTCs were used (the deposition numbers of NTCs are included in Additional file 4: Table S1), and the remaining 13 local tea cultivars (LTCs) were registered 
by the corresponding provincial government, while the subspecies type of four tea cultivars ('Keke 1', 'Keke 2', 'Ziyan' and 'Zixian') was still undetermined.

Young leaves of these tea cultivars were collected and immediately frozen in liquid nitrogen, and subsequently stored at $-80^{\circ} \mathrm{C}$ until further use. Total genomic DNA was extracted using the EZgene ${ }^{\mathrm{Tn}} \mathrm{CP}$ Plant Miniprep Kit (Biomiga, USA) following the manufacturer's protocol. The quality and quantity of DNA samples were determined by $1 \%$ agarose gel electrophoresis and the NanoDrop 2000 UV-Vis spectrophotometer, respectively. The concentration of each sample was adjusted to approximately $30 \mathrm{ng} / \mathrm{ul}$ for further use in the subsequent PCR amplifications.

\section{Identification of SNPs and InDels by genome-wide comparison}

Considering the quality of genome assemblies of 'Shuchazao' is better than the assemblies of 'Yunkang 10' [9, 10], it is reasonable to choose the assemblies of 'Shuchazao' as the reference genome. The clean reads of 'YunKang 10' were retrieved from the NCBI Sequence Read Archive under project number PRJNA381277 (Only the reads with library insert size equal to $500 \mathrm{bp}(\sim 10 \times)$ were applied for the further variation calling).

Subsequently, several steps were applied to identify genetic variations between the two assemblies: aligning the clean reads of 'Yunkang 10' to the reference using BWAMEM (version 0.7.17) with parameter '- $\mathrm{M}-\mathrm{R}-\mathrm{t} 40^{\prime}$ ', removing PCR duplicates with Picard program, calling SNPs and InDels using GATK-HaplotypeCaller method with parameter '-stand_call_conf 30', and the combination method of Samtools-mpileup with parameters '-ugf -t DP -t SP' and Bcftools-call with parametes ' $-\mathrm{v}-\mathrm{m}-\mathrm{O}$ ', respectively. Then take the intersection of the two results and use the GATK software to filter according to the following parameters: 'QD $<20.0||$ ReadPosRankSum $<-8.0||$ FS $>10.0||$ QUAL $<$ MMEANQUAL (the first filter)' and 'DP $<50.0|| \mathrm{GQ}<$ 10.0||QD $<20.0|| F S>200.0|| S O R \quad>10.0||$ MQRankSum $<-12.5||$ ReadPosRankSum <-8.0||QUAL < $\$$ MEANQUAL', finally get a high quality variation locus set (Additional file 1: Figure S1). Annotation for the remaining variations was conducted using snpEFF, and statistics of variations with Vcftools. The genes containing SNPs/InDels in CDs were selected by SnpSift, and their GO term enrichment analysis were performed using the free online platform OmicShare tools (http://www.omicshare.com/tools) (Additional file 1: Figure S1). These software programs have been accurately and expediently applied in SNP calling from nextgeneration sequencing data $[46,47]$.

\section{Validation and development of InDel markers}

To develop suitable InDel markers for genetic research, the InDel lengths $\geq 5$ and $\leq 20$ bp were used as candidate loci. Specific primers were designed based on the sequences flanking the InDel loci through the Primer 3.0 program with the following parameters: amplicons length (bp) 150-350; primer length 20-22, with the optimum length being $20 \mathrm{bp}$; $\operatorname{Tm}\left({ }^{\circ} \mathrm{C}\right) 50-60$, with $55^{\circ} \mathrm{C}$ being the optimum; GC content (\%) 40-60, with $50 \%$ being the optimum.

A total of 100 primer pairs were randomly selected and preliminarily screened on six tea cultivars ('Guyuxiang', 'Longjing 43', 'Echa 5', 'Guilv', Yungui', and 'Fudingdabaicha') using the Fragment Analyzer ${ }^{\mathrm{m}} 96$ (Advanced Analytical Technologies, Inc., Ames, IA). Primers that gave polymorphic and unambiguous bands were further screened for identification against the 46 tea cultivars. Details refer to PCR reagents and amplification conditions were performed according to our previous study [2]. If more than two fragments were amplified against some individuals using certain markers, only two fragments were collected based on the following criteria: selecting the higher peak value, the higher concentration of amplified products, and the more frequency of fragments occurred among other individuals.

\section{Genetic diversity analysis}

The PROSizeTM 2.0 included in the Fragment Analyzer ${ }^{\text {max }}$ 96 system was applied to visually select strong and clearly polymorphic DNA fragments for scoring, with the same strategy as described previously [8]. The values of expected heterozygosity $(\mathrm{He})$ and observed heterozygosity $(\mathrm{Ho})$ were determined by Popgene 32 version software. The number of alleles $(\mathrm{Na})$, major allele frequency (MAF), and polymorphism information content (PIC) were calculated using PowerMarker 3.25 [48]. Based on the PIC value, markers were divided into three types: highly informative (PIC $>0.5)$, moderately informative $(0.25<\mathrm{PIC}<$ $0.5)$ and slightly informative (PIC< 0.25$)$ [19].

\section{Population structure analysis}

Genetic structure analysis of distinct tea accessions was performed using the Structure 2.3.4 program [49]. To minimize Hardy-Weinberg and linkage disequilibrium within each group, the model-based Bayesian clustering algorithm was employed to assign individuals to groups with a predetermined number $(K$, it represents the number of inferred populations). Ten independent runs for each $K$ ranging from 2 to 9 were employed and 10,000 iterations were conducted for estimation after a 10,000 iterations burn-in period [19]. Estimation of the subgroups and the best $K$ value was performed according to a previous study [50].

\section{Phylogenetic analysis}

Nei's genetic distances of the 46 tea cultivars based on 48 InDel markers were calculated using PowerMarker 
3.25. The dendrogram was constructed using the neighbor-joining (NJ) algorithm as implemented in MEGA 7.0 [51], with bootstrap values at the default setting of 1000 replicates. Pairwise gap deletion mode was employed to guarantee that the divergent domains could contribute to the topology of the tree [52].

\section{Detection of catechin content using HPLC}

The contents of catechin and caffeine were extracted and examined according to the previous study [53]. All samples were detected with three independent biological replicates and each independent sample was examined with two technical replicates. The content of $(+)$-Gallocatechin (GC), (+)-Gallocatechin gallate (GCG), (-)-Epicatechin (EC), (-)-Epicatechin gallate (ECG), (-)-Epigallocatechin gallate (EGCG), and caffeine were detected. The catechin biosynthesis pathways were established according to previous studies [41, 54-57]. The number of SNP/InDel within the catechin/caffeine biosynthesis-related genes was also identified based on the result of alignment and functional annotation.

\section{Supplementary information}

Supplementary information accompanies this paper at https://doi.org/10. 1186/s12864-019-6347-0.

Additional file 1: Figure S1. Flowchart diagram for identifying genome-wide genetic variations between 'Shuchazao' and 'Yunkang 10' and functional annotation.

Additional file 2: Figure S2. Functional categorization of the genes containing genetic variations within the CDs region. a Functional annotation of genes containing SNPs within in the CDs region. $\mathbf{b}$ Functional annotation of genes containing InDels within in the CDs region. These genes were categorized based on $\mathrm{GO}$ annotation, and the number of each category is shown based on biological process, cellular component and molecular function.

Additional file 3: Figure S3. Exhibition of transferability and polymorphism detected by the remaining $30 \mathrm{InDel}$ markers among 46 tea cultivars.

Additional file 4: Table S1. Detailed information for the 46 tea cultivars used in this study.

Additional file 5: Table S2. Primer sequences of 48 newly developed InDel markers.

\begin{abstract}
Abbreviations
AFLPs: Amplified fragment length polymorphisms; CAPS: Cleaved amplified polymorphic sequence; EST: Expressed sequence tag; He: Expected heterozygosity; Ho: Observed heterozygosity; InDels: Insertions/Deletions; ISSRs: Inter-simple sequence repeats; MAF: Major allele frequency; $\mathrm{Na}$ : Number of alleles; PIC: Polymorphism information content; RADseq: Restriction site-associated DNA sequencing; RAPDs: Random amplification of polymorphic DNAs; RFLPs: Restriction fragment length polymorphisms; SLAF-seq: Specific locus amplified fragment sequencing; SNPs: Single nucleotide polymorphisms; SSRs: Simple sequence repeat
\end{abstract}

\section{Acknowledgments}

The authors thank the other members of our groups for technical assistance and appreciate the anonymous reviewers for constructive comments on this manuscript.

\section{Authors' contributions}

SRL performed data analysis and manuscript drafting. YLA conducted DNA extraction, primer design, PCR amplification, and InDel marker validation. WT were involved in the identification and analysis of variation loci. XJQ and LS were involved in sample collection and data analysis. XBX and RG are involved in DNA extraction and PCR amplification. CLW conceived and designed the research. All authors read and approved the final manuscript.

\section{Funding}

This work was financially supported by the Key R\&D Program of China (2018YFD1000601), the Anhui Provincial Natural Science Foundation (1808085QC92), the China Postdoctoral Science Foundation (2017 M621991), the Natural Science Foundation of Anhui Provincial Department of Education (KJ2018A0131), and the National Natural Science Foundation of China

(31800585). The funding bodies had no role in the design of the study, collection, analysis, and interpretation of data, and in writing the manuscript.

\section{Availability of data and materials}

Most of the important data generated or analyzed during this study are included in the article and its supplementary information files. The other data and materials associated with the current study are available from the corresponding author on reasonable request.

\section{Ethics approval and consent to participate}

Not applicable.

\section{Consent for publication}

Not applicable.

\section{Competing interests}

The authors declare that they have no competing interests.

\section{Author details}

${ }^{1}$ State Key Laboratory of Tea Plant Biology and Utilization, Anhui Agricultural University, 130 Changjiang West Road, Hefei, China. ${ }^{2}$ Guangxi LuYI Institute of Tea Tree Species, 17 Jinji Road, Guilin, China. ${ }^{3}$ Department of Biotechnology, Russian Research Institute of Floriculture and Subtropical Crops, Sochi, Russia.

Received: 25 August 2019 Accepted: 28 November 2019

Published online: 05 December 2019

\section{References}

1. Yang CS, Wang X, Lu G, Picinich SC. Cancer prevention by tea: animal studies, molecular mechanisms and human relevance. Nat Rev Cancer. 2009; 9(6):429-39.

2. Liu SR, Liu HW, Wu AL, Hou Y, An YL, Wei CL. Construction of fingerprinting for tea plant (Camellia sinensis) accessions using new genomic SSR markers. Mol Breed. 2017;37:93.

3. Zhang XC, Wu HH, Chen LM, Liu LL, Wan XC. Maintenance of mesophyll potassium and regulation of plasma membrane $\mathrm{H}+$-ATPase are associated with physiological responses of tea plants to drought and subsequent rehydration. Crop J. 2018;6:611-20.

4. Hashimoto M, Takasi S. Morphological studies on the origin of the tea plant $\checkmark$, a proposal of one place of origin by cluster analysis. Jpn J Trop Agric. 1978;21:93-101.

5. Chen L, Yu FL, Tong QQ. Discussions on phylogenetic classification and evolution of section Thea. J Tea Sci. 2000:20:89-94.

6. Chang HT. Thea-a section of beveragial tea trees of the genus Camellia. Acta Sci Nat Univ Sunyats. 1981;1:87-99.

7. Yu FL. Discussion on the originating place and the originating center of tea plants. J Tea Sci. 1986;6(1):1-8.

8. Liu SR, An YL, Li FD, Li SJ, Liu LL, Zhou QY, Zhao SQ, Wei CL. Genome-wide identification of simple sequence repeats and development of polymorphic SSR markers for genetic studies in tea plant (Camellia sinensis). Mol Breed. 2018;38:59.

9. Wei C, Yang H, Wang S, Zhao J, Liu C, Gao L, Xia E, Lu Y, Tai Y, She G, et al. Draft genome sequence of Camellia sinensis var. sinensis provides insights into the evolution of the tea genome and tea quality. Proc Natl Acad Sci U S A. 2018;115(18):E4151-8. 
10. Xia EH, Zhang HB, Sheng J, Li K, Zhang QJ, Kim C, Zhang Y, Liu Y, Zhu T, Li W et al. The tea tree genome provides insights into tea flavor and independent evolution of caffeine biosynthesis. Mol Plant. 2017;10(6):866-77.

11. Chen L, Gao QK, Chen DM, Xu CJ. The use of RAPD markers for detecting genetic diversity, relationship and molecular identification of Chinese elite tea genetic resources [Camellia sinensis (L.)O. Kuntze] preserved in tea germplasm repository. Biodivers Conserv. 2005;14(6):1433-44.

12. Ni S, Yao MZ, Chen L, Zhao LP, Wang XC. Germplasm and breeding research of tea plant based on DNA marker approaches. Front Agric China. 2008;2(2):200-7.

13. Mukhopadhyay M, Mondal TK, Chand PK. Biotechnological advances in tea (Camellia sinensis [L.] O. Kuntze): a review. Plant Cell Rep. 2016;35:255-87.

14. Hu YY, Mao BG, Peng Y, Sun YD, Pan YL, Xia YM, Sheng XB, Li YK, Tang $L$, Yuan $L P$, et al. Deep re-sequencing of a widely used maintainer line of hybrid rice for discovery of DNA polymorphisms and evaluation of genetic diversity. Mol Gen Genomics. 2014;289(3):303-15.

15. Garrido-Cardenas JA, Mesa-Valle C, Manzano-Agugliaro F. Trends in plant research using molecular markers. Planta. 2018;247(3):543-57.

16. Villano C, Esposito S, Carucci F, lorizzo M, Frusciante L, Carputo D, Aversano R. High-throughput genotyping in onion reveals structure of genetic diversity and informative SNPs useful for molecular breeding. Mol Breed. 2019;39:5.

17. Liu B, Wang Y, Zhai W, Deng J, Wang H, Cui Y, Cheng F, Wang XW, Wu J. Development of InDel markers for Brassica rapa based on whole-genome re-sequencing. Theor Appl Genet. 2012;126:231-9.

18. Thakur O, Randhawa GS. Identification and characterization of SSR, SNP and InDel molecular markers from RNA-Seq data of guar (Cyamopsis tetragonoloba, L. Taub.) roots. BMC Genomics. 2018;19(1):951.

19. Zhang TF, Gu MF, Liu YH, LV YD, Zhou L, Lu HY, Liang SQ, Bao HB, Zhao H. Development of novel InDel markers and genetic diversity in Chenopodium quinoa through whole-genome re-sequencing. BMC Genomics. 2017;18:685.

20. Belaj A, de la Rosa R, Lorite IJ, Mariotti R, Cultrera NGM, Beuzón CR, González-Plaza JJ, Muñoz-Mérida A, Trelles O, Baldoni L. Usefulness of a new large set of high throughput EST-SNP markers as a tool for olive Germplasm collection management. Front Plant Sci. 2018;9:1320.

21. Zhang N, Zhang H, Ren Y, Chen L, Zhang J, Zhang L. Genetic analysis and gene mapping of the orange flower trait in Chinese cabbage (Brassica rapa L.). Mol Breed. 2019;39:76.

22. Sarkar D, Kundu A, Das D, Chakraborty A, Mandal NA, Satya P, Karmakar PG, Kar CS, Mitra J, Singh NK. Resolving population structure and genetic differentiation associated with RAD-SNP loci under selection in tossa jute (Corchorus olitorius L.). Mol Genet Genomics. 2019;294:479-92.

23. Ujihara T, Taniguchi F, Tanaka J, Hayashi N. Development of expressed sequence tag (EST)-based cleaved amplified polymorphic sequence (CAPS) markers of tea plant and their application to cultivar identification. J Agric Food Chem. 2011;59:1557-64.

24. Zhang CC, Wang LY, Wei K, Cheng H. Development and characterization of single nucleotide polymorphism markers in Camellia sinensis (Theaceae). Genet Mol Res. 2014;13(3):5822-31.

25. Fang WP, Meinhardt LW, Tan HW, Zhou L, Mischke S, Zhang D. Varietal identification of tea (Camellia sinensis) using nanofluidic array of single nucleotide polymorphism (SNP) markers. Hortic Res. 2014;1:14035.

26. Ma JQ, Huang L, Ma CL, Jin JQ, Li CF, Wang RK, Zheng HK, Yao MZ, Chen L. Large-scale SNP discovery and genotyping for constructing a high-density genetic map of tea plant using specific-locus amplified fragment sequencing (SLAF-seq). PLoS One. 2015;10(6):e0128798.

27. Yang H, Wei C-L, Liu H-W, Wu J-L, Li Z-G, Zhang L, Jian J-B, Li Y-Y, Tai Y-L, Zhang J, et al. Genetic divergence between Camellia sinensis and its wild relatives revealed via genome-wide SNPs from RAD sequencing. PLoS One. 2016;11(3):e0151424

28. Deng W-W, Han J, Fan Y, Tai Y, Zhu B, Lu M, Wang R, Wan X, Zhang Z-Z. Uncovering tea-specific secondary metabolism using transcriptomic and metabolomic analyses in grafts of Camellia sinensis and C oleifera. Tree Genet Genomes. 2018;14:23.

29. Guo Y, Zhu C, Zhao S, Zhang S, Wang W, Fu H, Li X, Zhou C, Chen L, Lin Y, et al. De novo transcriptome and phytochemical analyses reveal differentially expressed genes and characteristic secondary metabolites in the original oolong tea (Camellia sinensis) cultivar 'Tieguanyin' compared with cultivar 'Benshan'. BMC Genomics. 2019;20(1):265.

30. Han J, Lu M, Zhu B, Wang R, Wan X, Deng W-W, Zhang Z-Z. Integrated transcriptomic and phytochemical analyses provide insights into characteristic metabolites variation in leaves of 1-year-old grafted tea (Camellia sinensis). Tree Genet Genomes. 2019;15:58.

31. Zhu B, Chen LB, Lu M, Zhang J, Han J, Deng WW, Zhang ZZ. Caffeine content and related gene expression: novel insight into caffeine metabolism in Camellia plants containing low, Normal, and high caffeine concentrations. J Agric Food Chem. 2019;67(12):3400-11.

32. Jander G, Norris SR, Rounsley SD, Bush DF, Levin IM, Last RL. Arabidopsis mapbased cloning in the post-genome era. Plant Physiol. 2002;129(2):440-50.

33. Ramakrishna G, Kaur P, Nigam D, Chaduvula PK, Yadav S, Talukdar A, Singh NK, Gaikwad K. Genome-wide identification and characterization of InDels and SNPs in Glycine max and Glycine soja for contrasting seed permeability traits. BMC Plant Biol. 2018;18(1):141.

34. Bai H, Cao Y, Quan J, Dong L, Li Z, Zhu Y, Zhu L, Dong Z, Li D. Identifying the genome-wide sequence variations and developing new molecular markers for genetics research by re-sequencing a landrace cultivar of foxtail millet. PLoS One. 2013;8(9):e73514.

35. Zhang JZ, Liu SR, Hu CG. Identifying the genome-wide genetic variation between precocious trifoliate orange and its wild type and developing new markers for genetics research. DNA Res. 2016;23(4):403-14.

36. García-Lor A, Luro F, Navarro L, Ollitrault P. Comparative use of InDel and SSR markers in deciphering the interspecific structure of cultivated citrus genetic diversity: a perspective for genetic association studies. Mol Gen Genomics. 2012;287(1):77-94.

37. Steri M, Idda ML, Whalen MB, Orru V. Genetic variants in mRNA untranslated regions. Wiley Interdiscip Rev RNA. 2018:9(4):e1474.

38. Yao MZ, Ma CL, Qiao TT, Jin JQ, Chen L. Diversity distribution and population structure of tea germplasms in China revealed by EST-SSR markers. Tree Genet Genomes. 2012:8:205-20.

39. Tan LQ, Peng M, Xu LY, Wang LY, Chen SX, Zou Y, Qi GN, Cheng $H$. Fingerprinting 128 Chinese clonal tea cultivars using SSR markers provides new insights into their pedigree relationships. Tree Genet Genomes. 2015;11:90.

40. Liu TJ, Li YP, Zhou JJ, Hu CG, Zhang JZ. Genome-wide genetic variation and comparison of fruit-associated traits between kumquat (Citrus japonica) and Clementine mandarin (Citrus Clementina). Plant Mol Biol. 2018;96(4-5):493-507.

41. Jin JQ, Ma JQ, Yao MZ, Ma CL, Chen L. Functional natural allelic variants of flavonoid 3',5'-hydroxylase gene governing catechin traits in tea plant and its relatives. Planta. 2017;245(3):523-38.

42. Chen L, Zhou ZX. Variations of main quality components of tea genetic resources [Camellia sinensis (L.) O. Kuntze] preserved in the China national germplasm tea repository. Plant Foods Hum Nutr. 2005;60:31-5.

43. Jin JQ, Ma JQ, Ma CL, Yao MZ, Chen L. Determination of catechin content in representative Chinese tea germplasms. J Agric Food Chem. 2014;62:9436-41.

44. Jin JQ, Yao MZ, Ma CL, Ma JQ, Chen L. Natural allelic variations of TCS1 play a crucial role in caffeine biosynthesis of tea plant and its related species. Plant Physiol Biochem. 2016;100:18-26.

45. Yang YJ, Liang YR. Clonal tea cultivars in China. Shanghai: Shanghai Scientific and Technical Publishers; 2014.

46. Wright B, Farquharson KA, McLennan EA, Belov K, Hogg CJ, Grueber CE. From reference genomes to population genomics: comparing three reference-aligned reduced-representation sequencing pipelines in two wildlife species. BMC Genomics. 2019;20:453.

47. Zhao Y, Wang $K$, Wang WL, Yin TT, Dong WQ, Xu CJ. A high-throughput SNP discovery strategy for RNA-seq data. BMC Genomics. 2019;20:160.

48. Liu K, Muse SV. PowerMarker: an integrated analysis environment for genetic marker analysis. Bioinformatics. 2005;21:2128-9.

49. Pritchard JK, Stephens M, Donnelly P. Inference of population structure using multilocus genotype data. Genetics. 2000;155(2):945-59.

50. Evanno G, Regnaut S, Goudet J. Detecting the number of clusters of individuals using the software structure: a simulation study. Mol Ecol. 2005;14(8):2611-20.

51. Kumar S, Stecher G, Tamura K. MEGA7: molecular evolutionary genetics analysis version 7.0 for bigger datasets. Mol Biol Evol. 2016;33:1870-4.

52. Liu SR, Khan MRG, Li YP, Zhang JZ, Hu CG. Comprehensive analysis of $\mathrm{CCCH}$-type zinc finger gene family in citrus (Clementine mandarin) by genome-wide characterization. Mol Gen Genomics. 2014;289:855-72.

53. Liu S, Mi X, Zhang R, An Y, Zhou Q, Yang T, Xia X, Guo R, Wang X, Wei C. Integrated analysis of miRNAs and their targets reveals that miR319C/TCP2 regulates apical bud burst in tea plant (Camellia sinensis). Planta. 2019;250:1111-29.

54. Shi CY, Yang H, Wei CL, Yu O, Zhang ZZ, Jiang CJ, Sun J, Li YY, Chen Q, Xia $T$, et al. Deep sequencing of the Camellia sinensis transcriptome revealed candidate genes for major metabolic pathways of tea-specific compounds. BMC Genomics. 2011;12:131. 
55. Wu ZJ, Li XH, Liu ZW, Xu ZS. Zhuang J De novo assembly and transcriptome characterization: novel insights into catechins biosynthesis in Camellia sinensis. BMC Plant Biol. 2014;14:277.

56. Li CF, Zhu Y, Yu Y, Zhao QY, Wang SJ, Wang XC, Yao MZ, Luo D, Li X, Chen $L$, et al. Global transcriptome and gene regulation network for secondary metabolite biosynthesis of tea plant (Camellia sinensis). BMC Genomics. 2015;16:560

57. Wang YS, Xu YJ, Gao LP, Yu O, Wang XZ, He XJ, Jiang XL, Liu YJ, Xia T. Functional analysis of flavonoid $3^{\prime}, 5^{\prime}$-hydroxylase from tea plant (Camellia sinensis): critical role in the accumulation of catechins. BMC Plant Biol. 2014; 14:347.

\section{Publisher's Note}

Springer Nature remains neutral with regard to jurisdictional claims in published maps and institutional affiliations.

Ready to submit your research? Choose BMC and benefit from:

- fast, convenient online submission

- thorough peer review by experienced researchers in your field

- rapid publication on acceptance

- support for research data, including large and complex data types

- gold Open Access which fosters wider collaboration and increased citations

- maximum visibility for your research: over $100 \mathrm{M}$ website views per year

At BMC, research is always in progress.

Learn more biomedcentral.com/submissions 